\title{
On the regularity of the Hausdorff distance between spectra of perturbed magnetic Hamiltonians
}

August 30, 2011

\author{
Horia D. Cornean!1, Radu Purice 2
}

\begin{abstract}
We study the regularity properties of the Hausdorff distance between spectra of continuous Harper-like operators. As a special case we obtain Hölder continuity of this Hausdorff distance with respect to the intensity of the magnetic field for a large class of magnetic elliptic (pseudo)differential operators with long range magnetic fields.
\end{abstract}

\section{Introduction}

Analytic perturbation theory tells us that if $V$ is relatively bounded to $H_{0}$, then the spectrum of $H_{\lambda}=H_{0}+\lambda V$ is at a Hausdorff distance of order $|\lambda|$ from the spectrum of $H_{0}$. This property is not true for singular perturbations (like for example the magnetic perturbation coming from a constant field), neither in the discrete nor in the continuous case.

Maybe the first proof of spectral stability of discrete Harper operators with respect to the variation of the intensity $b \geq 0$ of the external magnetic field is due to Elliott 9 . The result is refined in [7] where it is shown that the gap boundaries are $\frac{1}{3}$-Hölder continuous in $b$. Later results by Avron, van Mouche and Simon [4, Helffer and Sjöstrand [13, 14, and Haagerup and Rørdam [11] pushed the exponent up to $\frac{1}{2}$. In fact they prove more, they show that the Hausdorff distance between spectra behaves like $\left|b-b_{0}\right|^{\frac{1}{2}}$. These results are optimal in the sense that the Hölder constant is independent of the length of the eventual gaps, and it is known that these gaps can close down precisely like $\left|b-b_{0}\right|^{\frac{1}{2}}$ near rational values of $b_{0}$ [14, 12. Note that Nenciu [27] proves a similar result for a much larger class of discrete Harper-like operators. Many other spectral properties of Harper operators can be found in a paper by Herrmann and Janssen [15.

In the continuous case, the stability of gaps for Schrödinger operators was first shown by Avron and Simon [3], and Nenciu [26]. In [16] a very general result is obtained for perturbations of the anisotropic Laplacean. In 2] spectral continuity is proven for a large class of Hamiltonians defined by elliptic symbols. Nenciu's result implicitly gives a $\frac{1}{2}$-Hölder continuity in $b$ for the Hausdorff distance between spectra. Then in [6] the Hölder exponent of gap edges was pushed up to $\frac{2}{3}$.

The first proof of Lipschitz continuity of gap edges for discrete Harper-like operators was given by Bellissard [5] (later on Kotani [19] extended his method to more general regular lattices and dimensions larger than two). Very recently a completely different proof was given in 8$]$.

Our main technical result in this paper is Theorem 1.1, extending a previous result of Nenciu [27] and asserting Hölder continuity of a specific order for a class of bounded self-adjoint operators having a locally integrable integral kernel satisfying a weighted Schur-Holmgren estimate (1.1). This result, combined with the magnetic quantization [20, 22, 24, and the associated magnetic pseudodifferential calculus developped in [23, 17, 18, 21, allow us to we prove Theorem 3.1] stating Hölder continuity of order $1 / 2$ of the spectrum of resolvents associated to a large class of elliptic Hamiltonians in a $B C^{\infty}$ magnetic field, with respect to the intensity of the magnetic field. The case of unbounded operators will be considered elsewhere.

\footnotetext{
${ }^{1}$ Department of Mathematical Sciences, Aalborg University, Fredrik Bajers Vej 7G, 9220 Aalborg, Denmark

${ }^{2}$ Institute of Mathematics 'Simion Stoilow' of the Romanian Academy, P. O. Box 1-764, RO-014700 Bucharest, Romania
} 


\subsection{The setting and the main result}

Consider the Hilbert space $L^{2}\left(\mathbb{R}^{d}\right)$ with $d \geq 2$. Let $\langle x\rangle:=\sqrt{1+|\mathbf{x}|^{2}}$ and let $\alpha \geq 0$. We consider bounded integral operators $T \in B\left(L^{2}\left(\mathbb{R}^{d}\right)\right)$ to which we can associate a locally integrable kernel $T\left(\mathbf{x}, \mathbf{x}^{\prime}\right)$ which is continuous outside the diagonal and obeys the following weighted Schur-Holmgren estimate:

$$
\|T\|_{1, \alpha}:=\max \left\{\sup _{\mathbf{x}^{\prime} \in \mathbb{R}^{d}} \int_{\mathbb{R}^{d}}\left|T\left(\mathbf{x}, \mathbf{x}^{\prime}\right)\right|\left\langle\mathbf{x}-\mathbf{x}^{\prime}\right\rangle^{\alpha} d \mathbf{x}, \sup _{\mathbf{x} \in \mathbb{R}^{d}} \int_{\mathbb{R}^{d}}\left|T\left(\mathbf{x}, \mathbf{x}^{\prime}\right)\right|\left\langle\mathbf{x}-\mathbf{x}^{\prime}\right\rangle^{\alpha} d \mathbf{x}^{\prime}\right\}<\infty .
$$

Let us denote the set of all these operators with $\mathcal{C}_{1, \alpha}$. When $\alpha=0$, we need to introduce a uniformity condition. Let $\chi$ be the characteristic function of the interval $[0,1]$ and define

$$
\mathbb{R}^{d} \times \mathbb{R}^{d} \ni\left(\mathbf{x}, \mathbf{x}^{\prime}\right) \mapsto \chi_{M}\left(\mathbf{x}, \mathbf{x}^{\prime}\right):=\chi\left(\left|\mathbf{x}-\mathbf{x}^{\prime}\right| / M\right), \quad M \geq 1 .
$$

If $T \in \mathcal{C}_{1,0}$ we denote by $T_{M}$ the operator given by the integral kernel $\chi_{M}\left(\mathbf{x}, \mathbf{x}^{\prime}\right) T\left(\mathbf{x}, \mathbf{x}^{\prime}\right)$. Then we define $\mathcal{C}_{\text {unif }}$ to be the subset of $\mathcal{C}_{1,0}$ consisting of operators obeying the estimate

$$
\lim _{M \rightarrow \infty}\left\|T-T_{M}\right\|_{1,0}=0 .
$$

Note that if we only consider kernels $T\left(\mathbf{x}, \mathbf{x}^{\prime}\right)$ which are dominated by $L^{1}$ functions of $\mathbf{x}-\mathbf{x}^{\prime}$, then $\mathcal{C}_{\text {unif }}=\mathcal{C}_{1,0}$.

For $T \in \mathcal{C}_{1, \alpha}$, we are interested in a family of Harper-like operators $\left\{T_{b}\right\}_{b \in \mathbb{R}}$ given by kernels of the form $e^{i b \varphi\left(\mathbf{x}, \mathbf{x}^{\prime}\right)} T\left(\mathbf{x}, \mathbf{x}^{\prime}\right)$ with $\varphi: \mathbb{R}^{d} \times \mathbb{R}^{d} \mapsto \mathbb{R}$ a continuous phase function satisfying the two properties:

$$
\varphi\left(\mathbf{x}, \mathbf{x}^{\prime}\right)=-\varphi\left(\mathbf{x}^{\prime}, \mathbf{x}\right) \quad \text { and } \quad\left|\varphi(\mathbf{x}, \mathbf{y})+\varphi\left(\mathbf{y}, \mathbf{x}^{\prime}\right)-\varphi\left(\mathbf{x}, \mathbf{x}^{\prime}\right)\right| \leq|\mathbf{x}-\mathbf{y}|\left|\mathbf{y}-\mathbf{x}^{\prime}\right| .
$$

Clearly, $\left\{T_{b}\right\}_{b \in \mathbb{R}} \subset \mathcal{C}_{1, \alpha}$.

The Hausdorff distance between two real compact sets $A$ and $B$ is defined as:

$$
d_{H}(A, B):=\max \left\{\sup _{x \in A} \inf _{y \in B}|x-y|, \sup _{y \in B} \inf _{x \in A}|x-y|\right\} .
$$

And here is our main technical result:

Theorem 1.1. Let $H$ be self-adjoint and consider a family of Harper-like operators $\left\{H_{b}\right\}_{b \in \mathbb{R}}$ as above. Then the map

$$
\mathbb{R} \ni b \mapsto d_{H}\left(\sigma\left(H_{b}\right), \sigma(H)\right) \in \mathbb{R}_{+}
$$

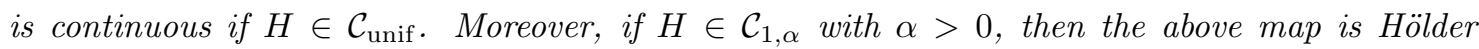
continuous with exponent $\beta:=\min \{1 / 2, \alpha / 2\}$. More precisely, for all $b_{0}$ we can find a constant $C>0$ such that:

$$
d_{H}\left(\sigma\left(H_{b_{0}+\delta}\right), \sigma\left(H_{b_{0}}\right)\right) \leq C|\delta|^{\beta} .
$$

Remark 1. Denoting by $\delta=b-b_{0}$, then according to our notations we have that $H_{b}=\left(H_{b_{0}}\right)_{\delta}$. It means that it is enough to prove the theorem at $b_{0}=0$.

Remark 2. It is natural to ask if the condition $H \in \mathcal{C}_{1, \alpha}$ is optimal in order to insure a Hölder continuity of order $\min \{1 / 2, \alpha / 2\}$; we believe in any case that if $\alpha$ becomes smaller and smaller, one cannot expect the Hölder coefficient to remain $1 / 2$. Similarly, if $\alpha=0$ it is unlikely to expect more than continuity of the Hausdorff distance. 


\section{Proof of Theorem 1.1}

Let $g \in C_{0}^{\infty}\left(\mathbb{R}^{d}\right)$ with $0 \leq g \leq 1, g(\mathbf{x})=1$ if $|\mathbf{x}| \leq 1 / 2$ and $g(\mathbf{x})=0$ if $|\mathbf{x}| \geq 2$. If $\mathbf{y} \in \mathbb{R}^{d}$, denote by $g_{\mathbf{y}}(\mathbf{x})=g(\mathbf{x}-\mathbf{y})$. By standard arguments, we may assume that $\sum_{\gamma \in \mathbb{Z}^{d}} g_{\gamma}^{2}(\mathbf{x})=1$ for all $\mathbf{x} \in \mathbb{R}^{d}$. For each $g_{\gamma}$ there is a finite number of neighbors whose supports are not disjoint from $\operatorname{supp}\left(g_{\gamma}\right)$, uniformly in $\gamma$.

Denote by $g_{\mathbf{y}, b}(\mathbf{x}):=g_{\mathbf{y}}\left(b^{1 / 2} \mathbf{x}\right)=g\left(b^{1 / 2} \mathbf{x}-\mathbf{y}\right)$. In this way we constructed a locally finite, quadratic partition of unity obeying

$$
\sum_{\gamma \in \mathbb{Z}^{d}} g_{\gamma, b}^{2}(\mathbf{x})=1, \quad \mathbf{x} \in \mathbb{R}^{d}
$$

and if $V_{\gamma, b}$ denotes the set of functions $g_{\gamma^{\prime}, b}$ whose supports are not disjoint from the support of $g_{\gamma, b}$, then $\sup _{\gamma \in \mathbb{Z}^{d}} \#\left\{V_{\gamma, b}\right\}$ is independent of $b$. Moreover, if $\chi_{\gamma, b}$ is the characteristic function of the support of $g_{\gamma, b}$ we have:

$$
\begin{aligned}
& \operatorname{supp}\left(g_{\gamma, b}\right) \subset\left\{\mathbf{x} \in \mathbb{R}^{d}:\left|\mathbf{x}-b^{-1 / 2} \gamma\right| \leq 2 b^{-1 / 2}\right\}, \\
& \left|g_{\gamma, b}(\mathbf{x})-g_{\gamma, b}(\mathbf{y})\right| \leq\||\nabla g|\|_{\infty}^{\epsilon} b^{\epsilon / 2}|\mathbf{x}-\mathbf{y}|^{\epsilon}\left\{\chi_{\gamma, b}(\mathbf{x})+\chi_{\gamma, b}(\mathbf{y})\right\}, \quad 0 \leq \epsilon \leq 1 .
\end{aligned}
$$

Lemma 2.1. Let $\left\{T_{\gamma}\right\}_{\gamma \in \mathbb{Z}^{d}} \subset B\left(L^{2}\left(\mathbb{R}^{d}\right)\right)$ possibly depending on $b$ such that

$$
\||T|\|_{\infty}:=\sup _{\gamma \in \mathbb{Z}^{d}}\left\|T_{\gamma}\right\|<\infty .
$$

Define on compactly supported functions the maps

$$
\psi \mapsto \Gamma(T)(\psi):=\sum_{\gamma \in \mathbb{Z}^{d}} \chi_{\gamma, b} T_{\gamma} \chi_{\gamma, b} \psi, \quad \tilde{\Gamma}(T)(\psi):=\sum_{\gamma \in \mathbb{Z}^{d}} \chi_{\gamma, b}\left|T_{\gamma} \chi_{\gamma, b} \psi\right| .
$$

Then both $\Gamma(T)$ and $\tilde{\Gamma}(T)$ can be extended by continuity to bounded maps on $L^{2}\left(\mathbb{R}^{d}\right)$ and there exists a constant $C$ independent of $b$ such that $\max \{\|\tilde{\Gamma}(T)\|,\|\Gamma(T)\|\} \leq C\|\| T \mid \|_{\infty}$.

Proof. Let $\psi \in L^{2}\left(\mathbb{R}^{d}\right)$ with compact support. We have:

$$
\begin{aligned}
\|\Gamma(T)(\psi)\|^{2} & \leq \sum_{\gamma \in \mathbb{Z}^{d}} \sum_{\gamma^{\prime} \in V_{\gamma, b}}\left|\left\langle\chi_{\gamma^{\prime}, b} T_{\gamma^{\prime}} \chi_{\gamma^{\prime}, b} \psi, \chi_{\gamma, b} T_{\gamma} \chi_{\gamma, b} \psi\right\rangle\right| \\
& \leq \sum_{\gamma \in \mathbb{Z}^{d} \gamma^{\prime} \in V_{\gamma, b}}\left\|T_{\gamma^{\prime}} \chi_{\gamma^{\prime}, b} \psi\right\|\left\|T_{\gamma} \chi_{\gamma, b} \psi\right\| \\
& \leq \frac{\left.\|T\|\right|_{\infty} ^{2}}{2} \sum_{\gamma \in \mathbb{Z}^{d}} \sum_{\gamma^{\prime} \in V_{\gamma, b}}\left(\left\|\chi_{\gamma^{\prime}, b} \psi\right\|^{2}+\left\|\chi_{\gamma, b} \psi\right\|^{2}\right) \leq C\|\mid\| T\|\|_{\infty}^{2}\|\psi\|^{2},
\end{aligned}
$$

where in the last inequality we used:

$$
\sum_{\gamma \in \mathbb{Z}^{d}} \sum_{\gamma^{\prime} \in V_{\gamma, b}}\left\|\chi_{\gamma^{\prime}, b} \psi\right\|^{2}=\int_{\mathbb{R}^{d}}|\psi(\mathbf{x})|^{2}\left\{\sum_{\gamma \in \mathbb{Z}^{d}} \sum_{\gamma^{\prime} \in V_{\gamma, b}} \chi_{\gamma^{\prime}, b}(\mathbf{x})\right\} d \mathbf{x} \leq C\|\psi\|^{2} .
$$

The same proof also works for $\tilde{\Gamma}(T)$ since the linearity is not used. Note that

$$
\left\|\tilde{\Gamma}(T)\left(\psi_{1}\right)-\tilde{\Gamma}(T)\left(\psi_{2}\right)\right\| \leq\left\|\tilde{\Gamma}(T)\left(\psi_{1}-\psi_{2}\right)\right\|
$$

which is enough for proving continuity. 
Lemma 2.2. Let $A$ be a positivity preserving bounded linear operator and define on compactly supported functions $\psi$ the following positively homogeneous map:

$$
\hat{\Gamma}_{A}(T)(\psi):=\sum_{\gamma \in \mathbb{Z}^{d}} \chi_{\gamma, b} A\left|T_{\gamma} \chi_{\gamma, b} \psi\right|
$$

Then $\hat{\Gamma}_{A}(T)$ can be extended by continuity to a bounded map on the whole space and $\left\|\hat{\Gamma}_{A}(T)\right\| \leq$ $C\|A\|\|T\|_{\infty}$.

Proof. We note that:

$$
\left|\hat{\Gamma}_{A}(T)\left(\psi_{1}\right)-\hat{\Gamma}_{A}(T)\left(\psi_{2}\right)\right| \leq \sum_{\gamma \in \mathbb{Z}^{d}} \chi_{\gamma, b} A\left|T_{\gamma} \chi_{\gamma, b}\left(\psi_{1}-\psi_{2}\right)\right|=\hat{\Gamma}_{A}(T)\left(\psi_{1}-\psi_{2}\right)
$$

due to the positivity preserving of $A$. Thus boundedness implies continuity. But the proof of Lemma 2.1 can be repeated almost identically, and the proof is over.

\subsection{The case $\alpha>0$}

If $z \in \rho(H)$, denote by $R(z)=(H-z)^{-1}$. We construct the operators

$$
T_{\gamma}(z):=e^{i b \varphi\left(\cdot, b^{-1 / 2} \gamma\right)} g_{\gamma, b} R(z) g_{\gamma, b} e^{-i b \varphi\left(\cdot, b^{-1 / 2} \gamma\right)}, \quad T(z):=\left\{T_{\gamma}(z)\right\}_{\gamma \in \mathbb{Z}}
$$

Then $\|\mid T(z)\|_{\infty} \leq 1 / \operatorname{dist}(z, \sigma(H))$. Introduce the notation

$$
f l\left(\mathbf{x}, \mathbf{y}, \mathbf{x}^{\prime}\right):=\varphi(\mathbf{x}, \mathbf{y})+\varphi\left(\mathbf{y}, \mathbf{x}^{\prime}\right)-\varphi\left(\mathbf{x}, \mathbf{x}^{\prime}\right) .
$$

The operator $\Gamma(T(z))$ is bounded (see Lemma 2.1). If Id denotes the identity operator, we can compute (use (2.1)):

$$
\left(H_{b}-z\right) \Gamma(T(z))=\mathrm{Id}+S(z)
$$

where

$$
\begin{aligned}
& (S(z) \psi)(\mathbf{x}) \\
& :=\sum_{\gamma \in \mathbb{Z}^{d}} e^{i b \varphi\left(\mathbf{x}, b^{-1 / 2} \gamma\right)} \int_{\mathbb{R}^{d}} d \mathbf{x}^{\prime} H\left(\mathbf{x}, \mathbf{x}^{\prime}\right)\left\{e^{i b f l\left(\mathbf{x}, \mathbf{x}^{\prime}, b^{-\frac{1}{2}} \gamma\right)}-1\right\} g_{\gamma, b}\left(\mathbf{x}^{\prime}\right)\left\{R(z) g_{\gamma, b} e^{-i b \varphi\left(\cdot, b^{-\frac{1}{2}} \gamma\right)} \psi\right\}\left(\mathbf{x}^{\prime}\right) \\
& +\sum_{\gamma \in \mathbb{Z}^{d}} e^{i b \varphi\left(\mathbf{x}, b^{-1 / 2} \gamma\right)} \int_{\mathbb{R}^{d}} d \mathbf{x}^{\prime} H\left(\mathbf{x}, \mathbf{x}^{\prime}\right)\left\{g_{\gamma, b}\left(\mathbf{x}^{\prime}\right)-g_{\gamma, b}(\mathbf{x})\right\}\left\{R(z) g_{\gamma, b} e^{-i b \varphi\left(\cdot, b^{-\frac{1}{2}} \gamma\right)} \psi\right\}\left(\mathbf{x}^{\prime}\right) \\
& =:\left(S_{1}(z) \psi\right)(\mathbf{x})+\left(S_{2}(z) \psi\right)(\mathbf{x}) .
\end{aligned}
$$

Let us analyze the contribution of the first term $\left(S_{1}(z) \psi\right)(\mathbf{x})$. Using the inequality (see also (1.4) )

$$
\left|e^{i b f l\left(\mathbf{x}, \mathbf{x}^{\prime}, b^{-\frac{1}{2}} \gamma\right)}-1\right| \leq 2^{1-\epsilon} b^{\epsilon}\left|\mathbf{x}-\mathbf{x}^{\prime}\right|^{\epsilon}\left|\mathbf{x}^{\prime}-b^{-\frac{1}{2}} \gamma\right|^{\epsilon}, \quad 0 \leq \epsilon \leq 1,
$$

we have:

$$
\begin{aligned}
& \left|S_{1}(z) \psi(\mathbf{x})\right| \\
& \leq 2^{1-\epsilon} b^{\epsilon} \int_{\mathbb{R}^{d}} d \mathbf{x}^{\prime}\left|H\left(\mathbf{x}, \mathbf{x}^{\prime}\right)\right|\left|\mathbf{x}-\mathbf{x}^{\prime}\right|^{\epsilon} \sum_{\gamma \in \mathbb{Z}^{d}} g_{\gamma, b}\left(\mathbf{x}^{\prime}\right)\left|\mathbf{x}^{\prime}-b^{-1 / 2} \gamma\right|^{\epsilon}\left|R(z) g_{\gamma, b} e^{-i b \varphi\left(\cdot, b^{-1 / 2} \gamma\right)} \psi\right|\left(\mathbf{x}^{\prime}\right) .
\end{aligned}
$$


With the notation $L_{\gamma}:=g_{\gamma, b}(\cdot)\left|\cdot-b^{-1 / 2} \gamma\right|{ }^{\epsilon} R(z) g_{\gamma, b} e^{-i b \varphi\left(\cdot, b^{-1 / 2} \gamma\right)}$ we see that the above inequality can be written as:

$$
\left|S_{1}(z) \psi(\mathbf{x})\right| \leq 2^{1-\epsilon} b^{\epsilon} \int_{\mathbb{R}^{d}} d \mathbf{x}^{\prime}\left|H\left(\mathbf{x}, \mathbf{x}^{\prime}\right)\right|\left|\mathbf{x}-\mathbf{x}^{\prime}\right|^{\epsilon}\{\tilde{\Gamma}(L) \psi\}\left(\mathbf{x}^{\prime}\right) .
$$

Using the fact that on the support of $g_{\gamma, b}$ we have $\left|\mathbf{x}^{\prime}-b^{-1 / 2} \gamma\right| \leq 2 b^{-1 / 2}$ it follows that $\||L|\|_{\infty} \leq$ $C b^{-\epsilon / 2}\|R(z)\|$, thus:

$$
\left\|S_{1}(z)\right\| \leq C \frac{b^{\epsilon / 2}}{\operatorname{dist}(z, \sigma(H))}\|H\|_{1, \epsilon} .
$$

Let us analyze the contribution from $S_{2}(z)$. Using (2.3) we can write:

$$
\begin{aligned}
& \left|S_{2}(z) \psi\right|(\mathbf{x}) \\
& \leq C b^{\epsilon / 2} \sum_{\gamma \in \mathbb{Z}^{d}} \int_{\mathbb{R}^{d}} d \mathbf{x}^{\prime}\left|H\left(\mathbf{x}, \mathbf{x}^{\prime}\right)\right|\left|\mathbf{x}-\mathbf{x}^{\prime}\right|^{\epsilon}\left\{\chi_{\gamma, b}(\mathbf{x})+\chi_{\gamma, b}\left(\mathbf{x}^{\prime}\right)\right\}\left|R(z) g_{\gamma, b} e^{-i b \varphi\left(\cdot, b^{-1 / 2} \gamma\right)} \psi\right|\left(\mathbf{x}^{\prime}\right) \\
& \leq C b^{\epsilon / 2} \sum_{\gamma \in \mathbb{Z}^{d}} \chi_{\gamma, b}(\mathbf{x}) \int_{\mathbb{R}^{d}} d \mathbf{x}^{\prime}\left|H\left(\mathbf{x}, \mathbf{x}^{\prime}\right)\right|\left|\mathbf{x}-\mathbf{x}^{\prime}\right|^{\epsilon}\left|R(z) g_{\gamma, b} e^{-i b \varphi\left(\cdot, b^{-1 / 2} \gamma\right)} \psi\right|\left(\mathbf{x}^{\prime}\right) \\
& +C b^{\epsilon / 2} \int_{\mathbb{R}^{d}} d \mathbf{x}^{\prime}\left|H\left(\mathbf{x}, \mathbf{x}^{\prime}\right)\right|\left|\mathbf{x}-\mathbf{x}^{\prime}\right|^{\epsilon} \sum_{\gamma \in \mathbb{Z}^{d}} \chi_{\gamma, b}\left(\mathbf{x}^{\prime}\right)\left|R(z) g_{\gamma, b} e^{-i b \varphi\left(\cdot, b^{-1 / 2} \gamma\right)} \psi\right|\left(\mathbf{x}^{\prime}\right)
\end{aligned}
$$

Now denoting with $A$ the operator with integral kernel $\left|H\left(\mathbf{x}, \mathbf{x}^{\prime}\right)\right|\left|\mathbf{x}-\mathbf{x}^{\prime}\right|^{\epsilon}$ and with $L_{\gamma}=$ $R(z) g_{\gamma, b} e^{-i b \varphi\left(\cdot, b^{-1 / 2} \gamma\right)}$ we obtain $\left|S_{2}(z) \psi\right| \leq C b^{\epsilon / 2}\left\{\hat{\Gamma}_{A}(L)(\psi)+A \tilde{\Gamma}(L)(\psi)\right\}$ thus

$$
\left\|S_{2}(z)\right\| \leq C \frac{b^{\epsilon / 2}}{\operatorname{dist}(z, \sigma(H))}\|H\|_{1, \epsilon}
$$

Going back to (2.6) we obtain the estimate:

$$
\|S(z)\| \leq C \frac{b^{\epsilon / 2}}{\operatorname{dist}(z, \sigma(H))}\|H\|_{1, \epsilon} .
$$

Now choose $0<\epsilon=\min \{\alpha, 1\}$. It follows that $\|S(z)\| \leq 1 / 2$ for every $z$ with $\operatorname{dist}(z, \sigma(H)) \geq$ $2 C b^{\epsilon / 2}\|H\|_{1, \epsilon}$, and by a standard argument it follows from (2.6) that $z \in \rho\left(H_{b}\right)$. Thus for every $x \in \sigma\left(H_{b}\right)$ we must have $\operatorname{dist}(x, \sigma(H)) \leq 2 C b^{\epsilon / 2}\|H\|_{1, \epsilon}$, thus

$$
\sup _{x \in \sigma\left(H_{b}\right)} \inf _{y \in \sigma(H)}|x-y| \leq 2 C b^{\min \{\alpha / 2,1 / 2\}}\|H\|_{1, \min \{\alpha, 1\}} .
$$

Now we can interchange $H_{b}$ with $H$ because

$$
H\left(\mathbf{x}, \mathbf{x}^{\prime}\right)=e^{-i b \phi\left(\mathbf{x}, \mathbf{x}^{\prime}\right)}\left\{e^{i b \phi\left(\mathbf{x}, \mathbf{x}^{\prime}\right)} H\left(\mathbf{x}, \mathbf{x}^{\prime}\right)\right\}=e^{-i b \phi\left(\mathbf{x}, \mathbf{x}^{\prime}\right)} H_{b}\left(\mathbf{x}, \mathbf{x}^{\prime}\right)
$$

and the $\|\cdot\|_{1, \alpha}$ norms are invariant with respect to the multiplication with a unimodular phase. Hence the Theorem is proved in the case $\alpha>0$.

\subsection{The case $\alpha=0$}

Due to our uniformity condition in (1.3) we can approximate $H_{b}$ in operator norm (uniformly in $b)$ with a sequence of operators $\left(H_{b}\right)_{M}$ which have strong localization near their diagonal. More precisely, given $\epsilon>0$ there exists $M=M(\epsilon)$ large enough such that $\left\|H_{b}-\left(H_{b}\right)_{M}\right\| \leq \epsilon / 3$ for every $b \in \mathbb{R}$. If $d\left(z, \sigma\left(H_{b}\right)\right)>\epsilon / 3$, then by writing

$$
\left(H_{b}\right)_{M}-z=\left[\mathrm{Id}-\left(H_{b}-\left(H_{b}\right)_{M}\right)\left(H_{b}-z\right)^{-1}\right]\left(H_{b}-z\right)
$$


it follows that $z \notin \sigma\left(\left(H_{b}\right)_{M}\right)$. It means that for every $x \in \sigma\left(\left(H_{b}\right)_{M}\right)$ we must have $d\left(x, \sigma\left(H_{b}\right)\right) \leq$ $\epsilon / 3$. By reversing the roles of $H_{b}$ and $\left(H_{b}\right)_{M}$ we conclude that $d_{H}\left(\sigma\left(H_{b}\right), \sigma\left(\left(H_{b}\right)_{M}\right)\right) \leq \epsilon / 3$, uniformly in $b \geq 0$. But now both $\left(H_{b}\right)_{M}$ and $H_{M}$ have strong localization near the diagonal, thus we can apply the result from $\alpha>0$, obtaining a $b(\epsilon)>0$ such that for every $|b| \leq b(\epsilon)$ we have $d_{H}\left(\sigma\left(H_{M}\right), \sigma\left(\left(H_{b}\right)_{M}\right)\right) \leq \epsilon / 3$. The proof is finished by the triangle inequality.

\section{Magnetic Hamiltonians}

Let us consider in $\mathbb{R}^{d}$ a magnetic field $B$ with components of class $B C^{\infty}\left(\mathbb{R}^{d}\right)$, i.e. bounded, smooth and with all its derivatives bounded. Consider a Hamiltonian given by a real elliptic symbol $h$ of class $S_{1}^{m}\left(\mathbb{R}^{d} \times \mathbb{R}^{d}\right)$ with $m>0$, i.e. $h \in C_{\mathrm{pol}}^{\infty}\left(\mathbb{R}^{d} \times \mathbb{R}^{d}\right)$ verifying the estimates:

$$
\begin{gathered}
\forall(a, \alpha) \in \mathbb{N}^{d} \times \mathbb{N}^{d}, \exists C(a, \alpha) \in \mathbb{R}_{+}, \quad \sup _{(x, \xi) \in \mathbb{R}^{d} \times \mathbb{R}^{d}}<\xi>|\alpha|-m\left|\left(\partial_{x}^{a} \partial_{\xi}^{\alpha} h\right)(x, \xi)\right| \leq C(a, \alpha), \\
\exists(R, C) \in \mathbb{R}_{+}^{2}, \quad|\xi| \geq R \Rightarrow h(x, \xi) \geq C|\xi|^{m}, \forall x \in \mathbb{R}^{d} .
\end{gathered}
$$

For our magnetic field $B$ we can choose a vector potential $A$ having components of class $C_{\mathrm{pol}}^{\infty}\left(\mathbb{R}^{d}\right)$; this can always be achieved by working with the transverse gauge:

$$
A_{j}(x):=-\sum_{k=1}^{d} \int_{0}^{1} d s B_{j k}(s x) s x_{k} .
$$

Let us denote by $\mathfrak{O p}^{A}(h)$ the magnetic quantization of $h$ defined as in 22. Then, this operator is self-adjoint on the magnetic Sobolev space $H_{A}^{m}\left(\mathbb{R}^{d}\right)$ and essentially self-adjoint on the space of Schwartz test functions (see Definition 4.2 and Theorem 5.1 in [17]). Moreover this operator is lower semibounded and satsfies a Gårding type inequality (Theorem 5.3 in [17]). Thus for any $\mathfrak{z} \in \mathbb{C} \backslash\left[-a_{0},+\infty\right)$, with $a_{0}>0$ large enough, we have that the following inverse exist

$$
\left(\mathfrak{O p}^{A}(h)-\mathfrak{z} \mathbb{1}\right)^{-1}=\mathfrak{O p}^{A}\left(r_{\mathfrak{z}}^{B}\right)
$$

and is defined by a symbol $r_{\mathfrak{z}}^{B}$ of class $S_{1}^{-m}\left(\mathbb{R}^{d} \times \mathbb{R}^{d}\right)$ (see Proposition 6.5 in [18). But using now Lemma A.4 in [23] and the fact that evidently $S_{1}^{m}\left(\mathbb{R}^{d} \times \mathbb{R}^{d}\right) \subset S^{m}\left(\mathbb{R}^{d} ; B C_{u}\left(\mathbb{R}^{d}\right)\right)$ with $S^{m}\left(\mathbb{R}^{d} ; B C_{u}\left(\mathbb{R}^{d}\right)\right)$ as in Definition A.3 of [23, (or Proposition 1.3.3 of [1]), we conclude that the symbol $r_{\mathfrak{z}}^{B}$ has a partial Fourier transform (with respect to the second variable) of class $L^{1}\left(\mathbb{R}^{d} ; B C_{u}\left(\mathbb{R}^{d}\right)\right)$. In fact looking closer to the proof of Lemma A.4 in 23] allows us to conclude (see also Proposition 1.3.6. in [1]) that the partial Fourier transform $\mathfrak{F}_{2}^{-1}\left[r_{\mathfrak{z}}^{B}\right](\mathbf{x}, \mathbf{y})$ has rapid decay in the second variable. Now, using formulas 3.28 and 3.29 in [22], we conclude that $\mathfrak{O p}^{A}\left(r_{\mathfrak{z}}^{B}\right)$ is an integral operator with kernel

$$
K^{A}\left(r_{\mathfrak{z}}^{B}\right)(\mathbf{x}, \mathbf{y}):=\left[\tilde{\Lambda}^{A} S^{-1}\left(\mathbf{1} \otimes \mathcal{F}_{2}^{-1}\right) r_{\mathfrak{z}}^{B}\right](\mathbf{x}, \mathbf{y})
$$

with

$$
\tilde{\Lambda}^{A}(\mathbf{x}, \mathbf{y}):=\exp \left\{-i \int_{\mathbf{x}}^{\mathbf{y}} A\right\}, \quad S^{-1}(\mathbf{x}, \mathbf{y}):=\left(\frac{\mathbf{x}+\mathbf{y}}{2}, \mathbf{x}-\mathbf{y}\right) .
$$

In conclusion:

$$
K^{A}\left(r_{\mathfrak{z}}^{B}\right)(\mathbf{x}, \mathbf{y})=\exp \left\{-i \int_{\mathbf{x}}^{\mathbf{y}} A\right\}\left\{\left[\mathbf{1} \otimes \mathcal{F}_{2}^{-1}\right] r_{\mathfrak{z}}^{B}\right\}\left(\frac{\mathbf{x}+\mathbf{y}}{2}, \mathbf{x}-\mathbf{y}\right) .
$$

Let us also notice that if we denote by $\langle 0, \mathbf{x}, \mathbf{y}\rangle$ the triangle of vertices $0, \mathbf{x}, \mathbf{y}$, we have that

$$
\left|\int_{\mathbf{x}}^{\mathbf{y}} A+\int_{\mathbf{y}}^{\mathbf{x}^{\prime}} A-\int_{\mathbf{x}}^{\mathbf{x}^{\prime}} A\right|=\left|\int_{<\mathbf{x}, \mathbf{y}, \mathbf{x}^{\prime}>} B\right| \leq\|B\|_{\infty}|\mathbf{x}-\mathbf{y}|\left|\mathbf{x}^{\prime}-\mathbf{y}\right|,
$$


with $\|B\|_{\infty}:=\max _{j, k} \sup _{\mathbf{x} \in \mathbb{R}^{d}}\left|B_{j, k}(\mathbf{x})\right|$.

Let us conclude that for any such magnetic field and elliptic symbol $h$, the resolvent $R=$ $\left(\mathfrak{O p}^{A}(h)+a\right)^{-1}$ is a bounded self-adjoint operator having a locally integrable integral kernel of the form $e^{i \varphi_{B}\left(\mathbf{x}, \mathbf{x}^{\prime}\right)} T_{B}\left(\mathbf{x}, \mathbf{x}^{\prime}\right)$ with

$$
\varphi_{B}\left(\mathbf{x}, \mathbf{x}^{\prime}\right):=-\int_{\mathbf{x}}^{\mathbf{x}^{\prime}} A, \quad T_{B}\left(\mathbf{x}, \mathbf{x}^{\prime}\right):=\left[S^{-1}\left(\mathbf{1} \otimes \mathcal{F}_{2}^{-1}\right) r_{\mathfrak{z}}^{B}\right]\left(\mathbf{x}, \mathbf{x}^{\prime}\right) .
$$

Now let us consider a magnetic field $B_{0}$ with components of class $B C^{\infty}\left(\mathbb{R}^{d}\right)$ and a small variation of it, in the same class, $B_{b}(\mathbf{x}):=B_{0}(\mathbf{x})+b \mathfrak{b}(\mathbf{x})$ with $b \in[0,1]$. Given an elliptic symbol $h$ as before we now have two Hamiltonians $H:=\mathfrak{O p}^{A_{0}}(h)$ and $H^{\prime}:=\mathfrak{O p}^{A}(h)$, with $A_{0}$ a vector potential for $B_{0}$ and $A$ a vector potential fos $B$. We can write $A(\mathbf{x})=A_{0}(\mathbf{x})+b \mathfrak{a}(\mathbf{x})$ with $\mathfrak{a}$ a vector potential for $\mathfrak{b}$. Then we have the following result:

Theorem 3.1. For $h, B_{0}$ and $B_{b}$ as above, consider $H=\mathfrak{O p}^{A_{0}}(h)$ and $H^{\prime}=\mathfrak{O p}^{A}(h)$. For $a>0$ large enough we define the two associated resolvents as above:

$$
\begin{aligned}
& R:=(H+a)^{-1}=\mathfrak{O p}^{A_{0}}\left(r_{-a}^{B_{0}}\right), \text { with integral kernel: } e^{-i\left[\int_{\mathbf{x}}^{\mathbf{x}^{\prime}} A_{0}\right]}\left[S^{-1}\left(\mathbf{1} \otimes \mathcal{F}_{2}^{-1}\right) r_{-a}^{B_{0}}\right]\left(\mathbf{x}, \mathbf{x}^{\prime}\right), \\
& R^{\prime}:=\left(H^{\prime}+a\right)^{-1}=\mathfrak{O p}^{A_{0}}\left(r_{-a}^{B_{0}}\right), \text { with integral kernel: } e^{-i\left[\int_{\mathbf{x}}^{\mathbf{x}^{\prime}} A\right]}\left[S^{-1}\left(\mathbf{1} \otimes \mathcal{F}_{2}^{-1}\right) r_{-a}^{B}\right]\left(\mathbf{x}, \mathbf{x}^{\prime}\right) .
\end{aligned}
$$

Then there exists a constant $C$ only depending on the symbol $h$ and on the magnetic field $B_{0}$ such that we have the following estimate:

$$
d_{H}\left(\sigma(R), \sigma\left(R^{\prime}\right)\right) \leq C \sqrt{b}
$$

Proof. Let us remark that the kernels $S^{-1}\left(\mathbf{1} \otimes \mathcal{F}_{2}^{-1}\right) r_{-a}^{B_{0}}$ and $S^{-1}\left(\mathbf{1} \otimes \mathcal{F}_{2}^{-1}\right) r_{-a}^{B}$ are the integral kernels of the operators given by the usual quantization (without magnetic field) $\mathfrak{O p}$ of the symbols $r_{-a}^{B_{0}}$ and resp. $r_{-a}^{B}$.

Proposition 3.2. Being symbols of negative order, both $r_{-a}^{B_{0}}$ and $r_{-a}^{B}$ define bounded operators on $L^{2}\left(\mathbb{R}^{d}\right)$ and we have that $\left\|\mathfrak{O} \mathfrak{p}\left(r_{-a}^{B_{0}}\right)-\mathfrak{O} \mathfrak{p}\left(r_{-a}^{B}\right)\right\|_{1,0} \leq C b$.

Proof. Using the ideas and results in 22 . we shall use the magnetic Moyal composition $\sharp^{B}$ defined by the quantization associated to the field $B$. Let us compute (as tempered distributions):

$$
(h+a) \sharp^{B} r_{-a}^{B_{0}}-1=(h+a) \sharp^{B} r_{-a}^{B_{0}}-(h+a) \sharp^{B_{0}} r_{-a}^{B_{0}}=: s_{-a}^{b} .
$$

Due to the general theory developped in [17, 18, $s_{-a}^{b}$ is defined by a symbol of class $S_{1}^{0}\left(\mathbb{R}^{d} \times \mathbb{R}^{d}\right)$ that can be computed by the following oscillating integral:

$$
\begin{gathered}
{\left[(h+a) \sharp^{B} r_{-a}^{B_{0}}-1\right](\mathbf{x}, \xi)=(2 \pi)^{-2 d} \int_{\Xi} \int_{\Xi} d \mathbf{y} d \eta d \mathbf{x}^{\prime} d \zeta} \\
\times e^{-2 i\left(\left\langle\mathbf{x}^{\prime}, \eta>-<\mathbf{y}, \zeta>\right)\right.}\left[\omega^{B}\left(\mathbf{x}, \mathbf{y}-\mathbf{x}, \mathbf{x}^{\prime}-\mathbf{x}\right)-\omega^{B_{0}}\left(\mathbf{x}, \mathbf{y}-\mathbf{x}, \mathbf{x}^{\prime}-\mathbf{x}\right)\right](h+a)(\mathbf{x}-\mathbf{y}, \xi-\eta) r_{-a}^{B_{0}}\left(\mathbf{x}-\mathbf{x}^{\prime}, \xi-\zeta\right) \\
=i b(2 \pi)^{-2 d} \int_{\Xi} \int_{\Xi} d \mathbf{y} d \eta d \mathbf{x}^{\prime} d \zeta e^{-2 i\left(<\mathbf{x}^{\prime}, \eta>-<\mathbf{y}, \zeta>\right)} \omega^{B_{0}}\left(\mathbf{x}, \mathbf{y}-\mathbf{x}, \mathbf{x}^{\prime}-\mathbf{x}\right) \theta_{b}\left(\mathbf{x}, \mathbf{y}-\mathbf{x}, \mathbf{x}^{\prime}-\mathbf{x}\right) \\
\times(h+a)(\mathbf{x}-\mathbf{y}, \xi-\eta) r_{-a}^{B_{0}}\left(\mathbf{x}-\mathbf{x}^{\prime}, \xi-\zeta\right),
\end{gathered}
$$

where

$$
\theta_{b}\left(\mathbf{x}, \mathbf{y}-\mathbf{x}, \mathbf{x}^{\prime}-\mathbf{x}\right)=e^{-i b \int_{<\mathbf{x}+\mathbf{y}-\mathbf{x}^{\prime}, \mathbf{x}+\mathbf{x}^{\prime}-\mathbf{y}, \mathbf{x}-\mathbf{y}-\mathbf{x}^{\prime}>^{\mathfrak{b}}}-1}
$$

is a function of class $B C^{\infty}\left(\mathbb{R}^{d} ; C_{\mathrm{pol}}^{\infty}\left(\mathbb{R}^{d} \times \mathbb{R}^{d}\right)\right)$ and we have the following estimates for its derivatives:

$$
\left|\left(\partial_{\mathbf{x}}^{\rho} \partial_{\mathbf{y}}^{\mu} \partial_{\mathbf{x}^{\prime}}^{\nu} \theta_{b}\right)\left(\mathbf{x}, \mathbf{y}-\mathbf{x}, \mathbf{x}^{\prime}-\mathbf{x}\right)\right| \leq C_{\rho, \mu, \nu} b^{1+\mid \rho+\mu+\nu}|\mathbf{y}|^{|\mu|}\left|\mathbf{x}^{\prime}\right|^{|\nu|}
$$


Now using Proposition 8.45 in [18] we conclude that $(h+a) \sharp^{B} r_{-a}^{B_{0}}-1$ is a symbol of type $S_{1}^{0}\left(\mathbb{R}^{d} \times \mathbb{R}^{d}\right)$ with seminorms of order at least $b$ and using Remark 3.3 in [17. we conclude that it defines a bounded operator with norm of order $b$. Thus for $b$ small enough we can invert $1+s_{-a}^{b}$ and obtain that (using once again Proposition 8.45 in [18] and the Calderon-Vaillancourt Theorem [10])

$$
\begin{gathered}
r_{-a}^{B}=r_{-a}^{B_{0} \sharp^{B}}\left\{1+s_{-a}^{b}\right\}^{-B}, \quad r_{-a}^{B}-r_{-a}^{B_{0}}=-r_{-a}^{B} \sharp^{B} s_{-a}^{b}, \\
\left\|\mathfrak{O p}\left(r_{-a}^{B_{0}}\right)-\mathfrak{O} \mathfrak{p}\left(r_{-a}^{B}\right)\right\|_{1,0} \leq C b .
\end{gathered}
$$

Now we shall consider the bounded self-adjoint operators $R_{b}$ with the kernel $e^{-i\left[\int_{\mathbf{x}}^{x^{\prime}} A\right]}\left[S^{-1}(\mathbf{1} \otimes\right.$ $\left.\left.\mathcal{F}_{2}^{-1}\right) r_{-a}^{B_{0}}\right]\left(\mathbf{x}, \mathbf{x}^{\prime}\right)$. Due to the above Proposition, by replacing $R^{\prime}$ with $R_{b}$ we make an error of order $b$ in operator norm on $L^{2}\left(\mathbb{R}^{d}\right)$. Now we see that $R_{b}$ is a Harper-type family, for which we can apply the results of Section 2. Here, $R=R_{0}$. We note that the integral kernels of $R_{b}$ have a common factor independent of $b$ which is of class $C_{1, \alpha}$ for any $\alpha \geq 0$. Moreover, the integral kernels of $R_{b}$ only differ by a unimodular exponential factor $e^{i b \varphi\left(\mathbf{x}, \mathbf{x}^{\prime}\right)}$ where $\varphi\left(\mathbf{x}, \mathbf{x}^{\prime}\right):=-\int_{\mathbf{x}}^{\mathbf{x}^{\prime}} \mathfrak{a}$ satisfies (1.4).

Therefore Theorem 1.1 implies that $d_{H}\left(\sigma\left(R_{b}\right), \sigma(R)\right) \leq C \sqrt{b}$, and since $d_{H}\left(\sigma\left(R_{b}\right), \sigma\left(R^{\prime}\right)\right) \leq$ $C b$ it follows that

$$
d_{H}\left(\sigma\left(R^{\prime}\right), \sigma(R)\right) \leq C \sqrt{b},
$$

which finishes the proof of the theorem.

Acknowledgments. H.C. acknowledges support from the Danish F.N.U. grant Mathematical Physics, and thanks Gheorghe Nenciu for many fruitful discussions. R.P. acknowledges support from CNCSIS grant PCCE 8/2010 Sisteme diferentiale in analiza neliniara si aplicatii, and thanks Aalborg University for hospitality.

\section{References}

[1] W.O. Amrein, A. Boutet de Monvel and V. Georgescu: $C_{0}$-Groups, Commutator Methods and Spectral Theory of N-Body Hamiltonians, Birkhäuser Verlag, 1996.

[2] N. Athmouni, M. Măntoiu, and R. Purice: On the continuity of spectra for families of magnetic pseudodifferential operators. Journal of Mathematical Physics 51, 083517 (2010); doi:10.1063/1.3470118 (15 pages).

[3] Avron, J.E., Simon, B.: Stability of gaps for periodic potentials under variation of a magnetic field. J. Phys. A: Math. Gen. 18, 2199-2205 (1985)

[4] Avron, J., van Mouche, P.H.M., Simon, B.: On the measure of the spectrum for the almost Mathieu operator. Commun. Math. Phys. 132, 103-118, (1990). Erratum in Commun. Math. Phys. 139, 215 (1991)

[5] Bellissard, J.: Lipshitz Continuity of Gap Boundaries for Hofstadter-like Spectra. Commun. Math. Phys. 160, 599-613 (1994)

[6] Briet, P., Cornean, H.D.: Locating the spectrum for magnetic Schrödinger and Dirac operators. Comm. Partial Differential Equations 27 no. 5-6, 1079-1101 (2002)

[7] Choi, M.D., Elliott, G.A., Yui, N.: Gauss polynomials and the rotation algebra. Invent. Math. 99, 225-246 (1990)

[8] Cornean, H.D.: On the Lipschitz continuity of spectral bands of Harper-like and magnetic Schrödinger operators. Ann. Henri Poincaré 11, 973-990 (2010) 
[9] Elliott, G.: Gaps in the spectrum of an almost periodic Schrodinger operator. C.R. Math. Rep. Acad. Sci. Canada 4, 255-259 (1982)

[10] Folland, B.G.: Harmonic Analysis in Phase Space. Annals of Mathematics Studies. Princeton University Press, 1989.

[11] Haagerup, U., Rørdam, M.: Perturbations of the rotation $C^{*}$-algebras and of the Heisenberg commutation relation. Duke Math. J. 77, 627-656 (1995)

[12] Helffer, B., Kerdelhue, P., Sjöstrand, J.: Mémoires de la SMF, Série 2 43, 1-87 (1990)

[13] Helffer, B., Sjöstrand, J.: Equation de Schrödinger avec champ magnétique et équation de Harper. Springer Lecture Notes in Phys. 345, 118-197 (1989)

[14] Helffer, B., Sjöstrand, J.: Analyse semi-classique pour l'équation de Harper. II. Bull. Soc. Math. France 117, Fasc. 4, Memoire 40 (1990)

[15] Herrmann, D.J.L., Janssen, T.: On spectral properties of Harper-like models. J. Math. Phys. 40 (3), 1197 (1999)

[16] V. Iftimie: Opérateurs differentiels magnétiques: Stabilité des trous dans le spectre, invariance du spectre essentiel et applications, Commun. in P.D.E. 18, 651-686, (1993).

[17] Iftimie, V., Măntoiu, M., Purice, R.: Magnetic Pseudodifferential Operators. Publications of the Research Institute for Mathematical Sciences 43 (3), 585-623 (2007)

[18] Iftimie, V., Măntoiu, M., Purice, R.: Commutator Criteria for Magnetic Pseudodifferential Operators. Communications in Partial Differential Equations 35, 10581094, (2010).

[19] Kotani, M.: Lipschitz continuity of the spectra of the magnetic transition operators on a crystal lattice. J. Geom. Phys. 47 (2-3), 323-342 (2003)

[20] Karasev, M.V. and Osborn, T.A.: Symplectic areas, quantization and dynamics in electromagnetic fields, J. Math. Phys. 43 (2), 756-788, 2002.

[21] Lein, M., Măntoiu, M., Richard, S.: Magnetic pseudodifferential operators with coefficients in $C^{*}$-algebras. http://arxiv.org/abs/0901.3704v1 (2009)

[22] Măntoiu, M., Purice, R.: The magnetic Weyl calculus. J. Math. Phys. 45 (4), 1394-1417 (2004)

[23] Măntoiu, M., Purice, R., Richard, S.: Spectral and propagation results for magnetic Schrodinger operators; A $C^{*}$-algebraic framework. J. Funct. Anal. 250 (1), $42-67$ (2007)

[24] Mantoiu, M.; Purice, R.; Richard, S.: Twisted crossed products and magnetic pseudodifferential operators. Advances in operator algebras and mathematical physics, 137-172, Theta Ser. Adv. Math., 5, Theta, Bucharest, 2005.

[25] Nenciu, G.: On asymptotic perturbation theory for quantum mechanics: almost invariant subspaces and gauge invariant magnetic perturbation theory. J. Math. Phys. 43 (3), 1273$1298(2002)$

[26] Nenciu, G.: Stability of energy gaps under variation of the magnetic field. Lett. Math. Phys. 11, 127-132 (1986)

[27] Nenciu, G.: On the smoothness of gap boundaries for generalized Harper operators. Advances in operator algebras and mathematical physics, Theta Ser. Adv. Math. 5, 173-182, Theta, Bucharest, 2005. arXiv:math-ph/0309009v2 